\title{
Partition dependence in development: Are children's decisions shaped by the arbitrary grouping of options?
}

\author{
Sheri Reichelson, Alexandra Zax, Andrea L. Patalano, \& Hilary C. Barth \\ Wesleyan University
}

Accepted for publication, Quarterly Journal of Experimental Psychology

This copy: Preprint 1.0, April 2018

\section{Word Count: 4520}

Corresponding author: Hilary C. Barth, Department of Psychology, Wesleyan University, 207 High St, Middletown, CT 06459, hbarth@wesleyan.edu

Author Note: We thank the participating families; the Connecticut Science Center; KidCity Children's Museum, Wadsworth Mansion and the Town of Middletown, CT; and lab members Aime Arroyo-Ramirez, Kerry Brew, Sarah Corner, Jamie Hom, Meghana Kandlur, Ilana Ladis, Maxine Lai, Jordan Feingold-Link, Praise Owoyemi, Joanna Paul, Elizabeth Shackney, and Katherine Williams. We also thank Craig Fox and Rebecca Ratner for helpful input. This work benefited from a Stipend Award from the National Living Laboratory Initiative with funding from NSF under Award Number 1113648, a Wesleyan University Project Grant, NSF DRL0950252 , and NSF DRL-1561214. 


\begin{abstract}
The grouping of options into arbitrary categories influences adults' decisions about allocating choices or resources among those options; this is called "partition dependence." Partition dependence has been demonstrated in a wide range of contexts in adults, and is often presented as a technique for designing choice architectures that nudge people toward better decisions. Whether children also make partition dependent decisions is unknown, as are potential patterns of developmental change. In this experiment $(N=159)$, we examined whether children exhibit partition dependence using a novel resource allocation task. This novel task, distributing food tokens to zoo animals, did elicit partition dependence in our developmental sample. Both older children (ages 7-10 years), and younger children (ages 3-6 years) made partition dependent allocations, and younger children exhibited a larger partition dependence effect than did older children. This work provides the first evidence that children's decisions, like adults', are influenced by the arbitrary grouping of the options, and suggests that younger children may be more susceptible to this influence, at least in the context explored here.
\end{abstract}

Keywords: partition dependence, diversification bias, decision making, cognitive development 
Partition dependence in development:

Are children's decisions shaped by the arbitrary grouping of options?

Some decisions involve choosing multiple options from a set of possibilities (e.g. buying yogurts at the grocery store). In such cases, the subjective or arbitrary groupings of the options influence adults' decisions: a phenomenon called partition dependence (e.g. Fox \& Rottenstreich, 2003). For example, imagine a hypothetical shopper intending to purchase 12 yogurts, with available options of strawberry, vanilla, and coffee. People presented with such situations tend to make diverse selections (selecting a variety of yogurt flavors, perhaps choosing four of each kind); this is called diversification bias (Simonson, 1990; see also Simonson \& Winer, 1992; Read, Antonides, van den Ouden, \& Trienekens, 2001). Because people may diversify over arbitrary groupings of options, not only over individual options, diversification bias can lead to partition dependence. That is, if our hypothetical shopper subjectively partitioned the set of available yogurts into "fruit" and "nonfruit" categories, they would be more likely to allocate their choices evenly over those two categories - selecting strawberry yogurts as half of the total, and vanilla and coffee yogurts together as the other half. Partitioning in this situation therefore leads to choices of more individual ungrouped items (strawberry yogurts) and fewer individual grouped items (vanilla or coffee yogurts).

Partition dependence in consumer choice has been experimentally demonstrated in many lab-based adult studies, and field evidence consistent with partition dependent behavior has also been widely observed (Fox, Bardolet, \& Lieb, 2005). In one study, participants were instructed to select several choice preferences from an array of wine descriptions. They diversified over country of origin when the descriptions were grouped by country, but over type of grape when the wines were grouped by grape, resulting in different choices across conditions (Fox, Ratner, \& Lieb, 2005). Partition dependence also arises in a range of situations beyond consumer choice. Many studies involve resource allocation decisions in which, rather than choosing options for consumption or use, the decision maker has a resource (e.g., time, money) to allocate among options. In a classic study, Benartzi and Thaler (2001) found that employees with 401(k) plans diversify, distributing retirement resources evenly over options in the plan. Further, partitioning options influences how adults diversify. In a task in which students were asked to allocate several free lunches over an academic year, Fox et al. (2005) showed that subdividing the year in different ways influenced responses.

Partition dependence has also been observed in predictions about the frequencies of properties of visually presented stimuli (See, Fox, \& Rottenstreich, 2006), weather predictions (Fox \& Rottenstreich, 2003), and market prices (in both lab and field studies; Sonnemann, Camerer, Fox, \& Langer, 2013). Bardolet, Fox, and Lovallo (2011) found lab and field evidence of partition dependence in corporate capital allocation decisions; Weber, Eisenführ, and von Winterfeldt (1988) found partition dependence in attribute weighting; and Fox and Levav (2004) found it in naïve judgments of conditional probabilities. Finally, the influence of partitions on decision making reaches beyond cases of diversification bias in which multiple selections or resources must be allocated among possible options. In a recent vignette-based study, the prescribing behavior of health-care providers was biased by the arbitrary partitioning of available options even though they were selecting only one item (Tannenbaum et al., 2015; see also Tannenbaum, Fox, \& Goldstein, n.d.). Partition dependence therefore has been demonstrated in many contexts in adults, and continues to be explored as a technique that choice architects can 
use to nudge people toward desired decisions (e.g. Johnson et al., 2012; Thaler \& Sunstein, 2008. However, it is unknown whether children also exhibit partition dependence in decision making.

Recently there has been increased interest in the evolutionary and developmental origins of various biases in decision making. Comparative and developmental researchers argue that questions of origin are crucial for a full understanding of human decision behavior. Studies with children and nonhuman primates elucidate the cognitive mechanisms underlying particular biases, and the experiences necessary for adult decision patterns to emerge (Harbaugh \& Krause, 2002; Santos \& Rosati, 2015). For example, framing effects (differing responses when decisions are framed in terms of losses vs. gains) don't depend on capacities unique to mature humans or experiences with economic markets, because they appear in children and capuchins (Lakshminarayanan et al, 2011; Levin \& Hart, 2003; Santos \& Rosati, 2015; Schlottman \& Tring, 2005; cf. Reyna \& Ellis, 1994).

Investigations of the developmental and evolutionary origins of partition dependence may be able to shed light on cognitive mechanisms and necessary experiences underlying the phenomenon. For example, we can ask whether experience with economic markets or formal decision situations is required for the emergence of partition dependent behavior by testing for such behavior in children. But partition dependence research with children faces a challenge: many existing paradigms are poorly suited for use with young populations, often employing verbal descriptions of hypothetical situations that are too complex for children. One exception is the simple consumer choice task of Fox et al., 2005 (Experiment 4), in which adult participants chose from a set of physical options that were perceptually partitioned. In this task, participants chose 5 individual candies from 4 types of candy arranged across 3 bowls, such that evidence of partition dependent choice appears if selections are influenced by the arbitrary partitioning of candy types into bowls). Previous work in our lab used the candy choice paradigm to test for partition dependence in children's choices. However, not only did we fail to find evidence of partition dependent choice in children, but in three experiments we did not replicate the previously reported findings of adult partition dependence in this task (Reichelson, Zax, Bass, Patalano, \& Barth, 2017). This work shows that the candy choice task itself does not reliably elicit partition dependence, even in adults - leaving open the question of whether partition dependence in decision making occurs in children.

There are at least three reasons to think that children's choices, like adults', might be influenced by the groupings of options when children are tested with age-appropriate versions of paradigms that do elicit partition dependence reliably in adults. First, there is evidence that children diversify across options like adults (e.g. Read \& Loewenstein, 1995), so at least one strategy leading to partition dependence - diversification bias - appears present in childhood (see also Echelbarger \& Gelman, 2017). Second, children as young as four flexibly reason about entities belonging to multiple categories (see Nguyen \& Girgis, 2014, for review), so children might draw on category groupings rather than concrete choice options when employing a diversification strategy. Third, partition dependence is more pronounced among naïve decision makers (compared to experts; Fox et al., 2005; Fox \& Clemen, 2005), and children can reasonably be said to fall into the former category: this implies not only that children could be susceptible to partition dependence effects, but also that younger children might show a stronger effect than older children. However, it is also possible that partition dependence might appear late in childhood, or even in adolescence or adulthood. Most findings of partition dependence concern complex, formal decision situations. Perhaps partition dependence is less likely in the decision situations available to children (an idea that finds some support in the repeated lack of 
partition dependence in both children's and adults' choices in candy choice tasks; Reichelson et al., 2017). It is also possible that attention to higher order partitions (vs. individual choice options) doesn't occur early in life. Also, some mechanisms underlying partition dependence may be deliberative rather than spontaneous (Fox et al., 2005) and therefore perhaps less available to young children.

To effectively explore partition dependence in child populations, an appropriate paradigm must first be developed: to do so was one goal of the present work. In order to create such a paradigm, we incorporated both elements of the simple consumer choice task mentioned earlier and elements of resource allocations tasks used previously with adult samples. To give the necessary background for the logic of the design, we first describe a broader theoretical view of partition dependence.

Fox and colleagues (2005) characterized diverse partition dependent behaviors as a general cognitive phenomenon: when people must allocate some limited resource (choices, money, probabilities), over a set of possibilities (items for consumption, recipients, events), their final allocations represent some compromise between intrinsic preferences and general tendencies toward equal allocation. For example, in a hypothetical financial aid allocation task (Fox et al., 2005), adults decided how university funds should be allocated across six different income ranges. In the "low-income" condition, the six income categories ranged from $<\$ 15,000$ $>\$ 75,000$; in the "high-income" condition, categories ranged from $<\$ 75,000->\$ 145,000$.

Participants were explicitly instructed to allocate "in an equitable manner," and the task suggested a need-based norm, activating an intrinsic preference to give more to those with less. Indeed, more aid was allocated to lower-income families overall. Critically, though, an equality norm toward even distribution across available categories also influenced decisions: Participants allocated more financial aid to lower-income families when the lower end of the income range was partitioned into more distinct categories (vs. when the upper end was partitioned into more categories), even though the categories were explicitly described as arbitrary (Fox et al., 2005).

Here, we used a novel method to determine whether children would exhibit partition dependent behavior. We developed a child-friendly resource allocation task that shares some characteristics of both the consumer choice and resource allocation tasks that have previously been used to explore partition dependence. Participants were asked to help a puppet distribute twelve tokens representing treats to four different types of zoo animals arrayed across three boxes. Two boxes were each arbitrarily associated with one type of animal, while the third box was associated with two animals (Figure 1), to create an arbitrary three-way partition of containers and a four-way partition of specific elements associated with the containers (as in previous consumer choice studies, Fox et al., 2005; Reichelson et al., 2017). There were therefore two "single-animal boxes" and one "double-animal box," with counterbalancing of the specific animal types appearing across these box types.

Children might choose to allocate evenly across animal types, as implied by fairness norms intended to be evoked by the instructions (the puppet wants to be fair, and is giving the food to the animals). Prior work on the development of equality preferences suggests children may be expected to exhibit intrinsic preferences for equal allocation toward recipients (e.g. Fehr, Bernhard, \& Rockenbach, 2008; LoBue, Nishida, Chiong, DeLoache, \& Haidt, 2011; Smith, Blake, \& Harris, 2013). This would lead to roughly equal allocations across box types on average (approximately half to the two single-animal boxes combined, and half to the double-animal box). Children might alternatively choose to allocate tokens based on an intrinsic preference for a certain animal type, and (due to counterbalancing) on average this would also lead to roughly 
equal allocations across box types (approximately half to the two single-animal boxes combined, and half to the double-animal box). Critically, if there is an influence of the arbitrary 3-way box partition, allocations should be biased toward the single-animal boxes (because a fully box-based allocation strategy would result in two thirds of allocations going to the single-animal boxes combined, and one third going to the double-animal box). This influence of the arbitrary box partition would constitute evidence of partition dependence.

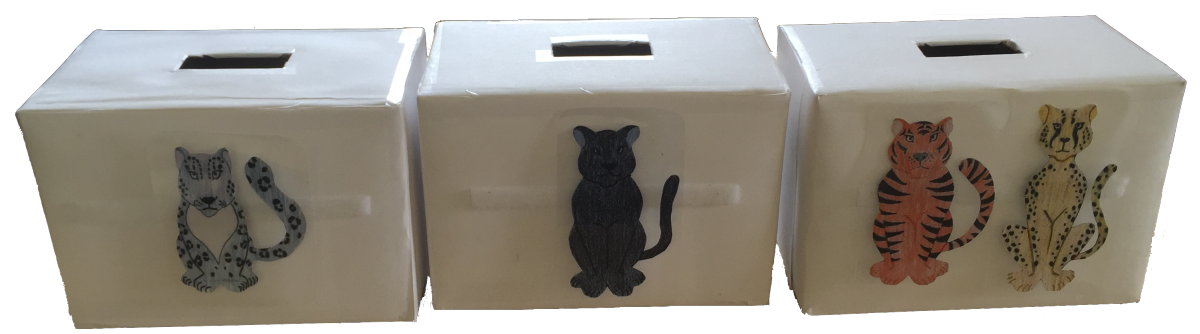

Figure 1.

Stimulus materials (food token boxes) used in the present experiment.

We administered the task to a wide developmental range (ages $3-11$ years) to achieve two main goals: to determine (1) whether partition dependence in decision making is present in childhood and (2) whether developmental change occurs in children's partition-dependent resource allocation. Children distributed tokens representing edible treats, with the design and predictions described above. Even young preschoolers who are not yet able to count can use turn-taking strategies to achieve equal allocations (e.g. Chernyak, Sandham, Harris, \& Cordes, 2016), so it is appropriate to include children with little or no formal mathematical knowledge in our sample. Given prior evidence that children use a diversification heuristic, that we have no a priori reason to expect great expertise or preference in this domain (which can reduce partition dependence; Fox et al., 2005), and that physical partitioning is not cognitively complex, we predicted that children would show partition dependence in this task by allocating more than half the tokens to the single-animal boxes. Although we did not make specific a priori predictions about the relationship between possible developmental changes and potential mechanisms underlying partition dependence, we did aim to set the stage for future developmental investigations of underlying mechanisms. For example, it has been proposed that under some circumstances, mechanisms giving rise to partition dependence are spontaneous and require little reflection, but that in other situations, they are more deliberative (Fox et al., 2005). These possibilities could be further distinguished by developmental work. Taking the first steps to develop a novel age-appropriate paradigm, establish whether partition dependence exists in children, and determine whether patterns of developmental change emerge is the purpose of the present study.

\section{Method}

Participants. One hundred and fifty-nine children $\left(M_{\mathrm{age}}=6\right.$ years and 11 months, range $=3$ years and 1 month -11 years and 9 months, 90 females) participated and were included in analyses. Twelve additional participants were excluded due to noncompliance, family interference, or experimenter error. 
Procedure. Children participated at a local science center (54\%), in our laboratory (35\%), at a children's museum $(6.3 \%)$, or at a town event $(4.4 \%)$. Children were first shown a genderneutral puppet, called "Alex", whom they were told had food treats for animals at the zoo. The researcher stated that Alex wanted to be fair (our adaptation of instructions in related adult allocation tasks that resources "must be distributed in an equitable manner," Fox et al, 2005), and asked the child to help Alex distribute the food.

Children were given twelve "food" tokens (2-inch wooden discs) in a bowl, and were told they could place the tokens in any of three white boxes $(22 \mathrm{~cm} \times 12 \mathrm{~cm} \times 15 \mathrm{~cm}$, with openings just large enough for the tokens) to indicate they would like to give them to different kinds of animals. The boxes were placed in a row facing the child. Two boxes were each marked with a single, distinct animal photo (single-animal boxes), and the third was marked with two distinct animal photos (double-animal box). Particular animals were assigned to boxes in front of the child to convey that box/animal associations were arbitrary, paralleling adult studies in which partition arbitrariness is stated explicitly. To do this, the experimenter said, "Hmmm ... let's put the food for all of the (animal type) at the zoo in this box!" while fixing animal photos to boxes. See Appendix A for a sample script for one condition.

Four photos of large cats (a leopard, cheetah, panther, and tiger) approximately the same size (about $80 \mathrm{~cm}^{2}$ ) were designed to be easily distinguished yet similar enough that children would be unlikely to have strong preferences. Photos were affixed to boxes with hook-and-loop fasteners. There were two possible physical arrangements of animal types and box orders (four counterbalancing conditions). Physical arrangements were: panther and cheetah on single animal boxes and leopard/tiger together on double-animal box, or leopard and tiger on single-animal boxes and panther/cheetah on double-animal box. Box orders were (L-R): single/single/double and double/single/single. Each animal appeared an equal number of times with each type of box and in each position. After tokens were allocated, children were asked which animal they preferred.

\section{Results}

Children distributed more than half the 12 tokens to single-animal boxes, $M=7.56, S D=$ $1.37, t(158)=14.39, p<.001, d=1.14$, two-tailed, consistent with partition dependence. Token distribution to single-animal boxes differed significantly between the single/single/double box order $(M=7.33, S D=1.47)$ and double/single/single box order $(M=7.77, S D=1.23) ; t(157)=$ $2.06, p=.041)$. The difference was not significant when the data were analyzed excluding the four participants (out of 159) who allocated 10 or more tokens to one box, $t(153)=-1.79, p=$ .076), thus this difference may not be best understood as a box order effect. Nevertheless, we tested for evidence of partition dependence in both box order conditions separately, and found that such evidence was present. Children in the single/single/double box order condition distributed more than half of the 12 tokens to single-animal boxes $(M=7.33, S D=1.47, t(75)=$ $7.86, p<.001, d=0.90$, two-tailed) and children in the double/single/single box order condition also did so $(M=7.77, S D=1.23, t(82)=13.09, p<.001, d=1.44$, two-tailed $)$.

We also examined the relationship between partition dependence and age. There was a negative correlation between tokens distributed to single-animal boxes and age in months, Pearson's $r(157)=-.26$, in the moderate effect size range, $p=.001$ (Figure 2). As age increased, the number of tokens allocated to single-animal boxes decreased: younger children's allocations appear more susceptible to partitioning effects. 


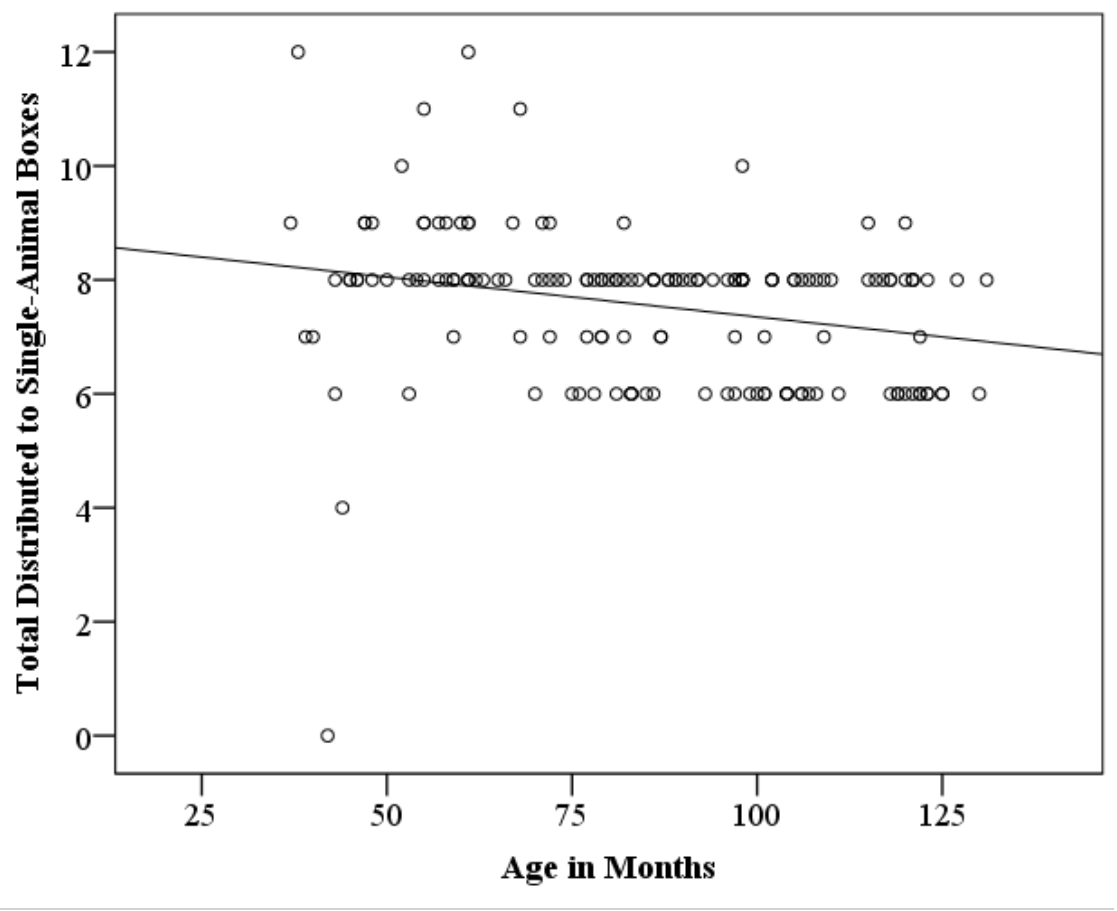

Figure 2.

A scatter plot of total tokens distributed to single-animal boxes as a function of age. Number of tokens distributed to single-animal boxes decreased as age increased $(r(157)=-.26, p=.001)$.

The data were then analyzed by splitting children into two roughly even groups by age in years: older children (7-10 years) and younger children (3-6 years). Older children ( $\mathrm{N}=82, M_{\text {age }}$ $=8 ; 8$, range $=7 ; 0-11 ; 9,51$ females $)$ distributed more than half the 12 tokens to single-animal boxes, $M=7.27, S D=1.02, t(81)=11.27, p<.001, d=1.24$, two-tailed, consistent with partition dependence (Figure 3 ). These results could not have been driven by allocation patterns inconsistent with diversification, such as putting all the tokens in one box: No older children allocated more than 8 tokens to a single box. Younger children $\left(\mathrm{N}=77, M_{\text {age }}=5 ; 3\right.$, range $=3 ; 1$ $6 ; 9,39$ females) also distributed more than half the 12 tokens to single-animal boxes overall, $M=$ $7.87, S D=1.61, t(76)=10.20, p<.001, d=1.16$, two-tailed (Figure 3 ). Some younger children allocated all 12 tokens to one box $(n=3)$, but with these children excluded, more than half the tokens were still distributed to single-animal boxes, $M=7.86, S D=1.17, t(73)=13.66, p<.001$, $d=1.59$, two-tailed; therefore extreme allocation patterns inconsistent with diversification did not produce the effect. (No children in the younger subsample allocated 11/12 tokens to singleanimal boxes, 1 child allocated 10/12, and no children allocated 9/12.) 

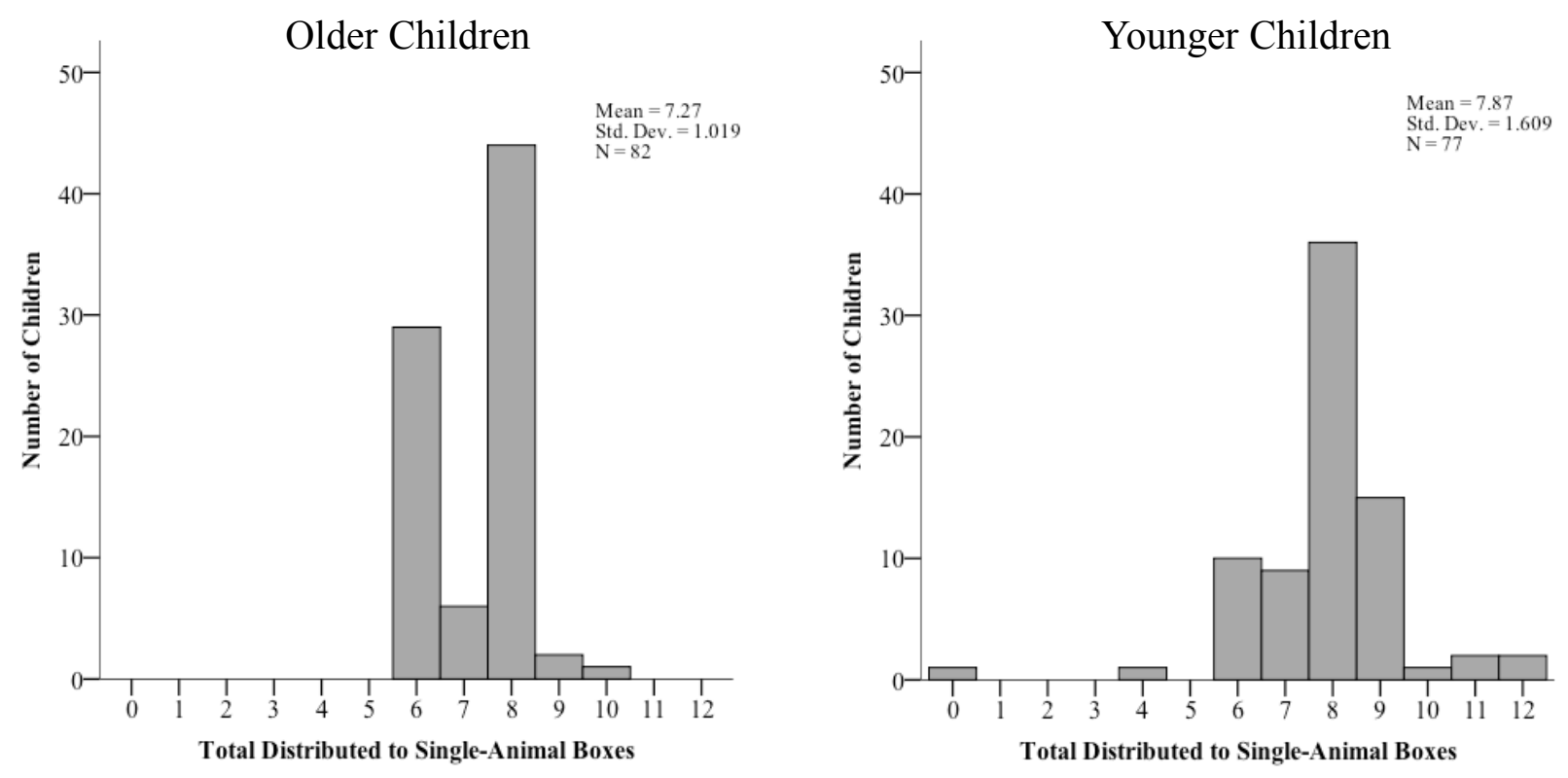

Figure 3.

Histograms of the number of tokens allocated to single-animal boxes by older children, ages 710 (left) and younger children, ages 3-6 (right). Evidence of partition dependence in this paradigm consists of a bias toward allocating more than half (more than 6) of the tokens to the single-animal boxes. The older subsample appears to exhibit a bimodal distribution with most children allocating 8 tokens to single-animal boxes (consistent with equal allocation across boxes), and a substantial minority allocating 6 tokens to single-animal boxes (consistent with equal allocation across animal types). The younger subsample appears to exhibit a unimodal distribution with most children allocating 8 tokens to single-animal boxes (consistent with equal allocation across boxes).

Overall, preschool- and elementary-school aged children demonstrated unambiguous partition dependence in this novel resource allocation task, and younger children exhibited greater partition dependence than older children.

\section{Discussion}

We investigated the phenomenon of partition dependence in children's decision making by developing and administering a novel resource allocation task. Children distributed tokens representing food to four different types of zoo animals, arrayed across three boxes. If the arbitrary 3-way box partition influenced children's allocations, children would allocate more tokens to two single-animal boxes than to one double-animal box. The allocations of both older children (aged 7-10 years) and younger children (aged 3-6 years) showed clear evidence of partition dependence. This finding constitutes the first evidence of partition dependence in children's decision making: the structuring of the available option categories influences the decisions of children, as well as adults.

These results extend prior findings that arbitrary groupings influence adults' resource allocations (e.g., Fox et al., 2005). That partition dependence was exhibited by children shows that it can emerge at a young age, rather than arising only from long experience with decisions or economic markets, or from considerable experience interpreting novel partitions of entities. 
Partition dependence was stronger for younger than for older children, consistent with previous evidence that adults who lack expertise in a domain are more likely to exhibit partition dependence (Fox et al., 2005).

As mentioned in the introduction, we did not make specific a priori predictions about which developmental patterns would be predicted by particular mechanisms that might give rise to partition dependence. However, the pattern of results we observed is suggestive as to the mechanisms that might underlie partition dependent allocations in this paradigm. Partition dependence is not thought to be a unitary phenomenon, but rather a broad cognitive phenomenon that can arise as a result of multiple specific mechanisms. Whenever people must allocate a limited resource over a set of possibilities, their allocations often represent some compromise between intrinsic preferences or norms and general tendencies toward equal allocation that can take multiple forms (Fox, Bardolet et al., 2005). It has been proposed that mechanisms giving rise to partition dependence in some paradigms may be spontaneous, requiring little reflection, while other such mechanisms may be deliberative such that partition dependence is the result of the overriding of more basic or intuitive tendencies (Fox et al., 2005). Given that younger children showed a larger partition dependence effect in the present study, it seems likely that the mechanisms underlying the effect in this particular paradigm are more spontaneous and less deliberative. To the extent that the underlying mechanisms are more spontaneous and less deliberative, we might expect them to have a greater influence on resource allocation in the developmental period when executive function and cognitive control processes are not fully developed. A related prediction is that partition dependence effects in this paradigm would be greater in adults performing the task under working memory load.

Children's behavior in resource allocation tasks can in some cases be influenced by their mathematics abilities. For example, in one recent study of preschoolers, older children were significantly more likely to distribute resources equally between two third-party recipients, and these age differences were explained by differences in children's mathematical abilities, specifically cardinal principle knowledge (Chernyak et al., 2016). Chernyak and colleagues also found that distribution strategies differed between children with more advanced cardinal principle knowledge (who divided resources in half first and then gave half to each receiver) and those with less advanced cardinal principle knowledge (who used a "turn-taking strategy" of handing out resources one at a time to the receivers). Though we did not explicitly test for the influences of children's mathematical abilities in the present study, it seems likely that they played a role. The multiplicative relations of the items in our stimulus set ( 3 boxes, 4 animal types, 12 tokens) were surely opaque to the youngest children, and more transparent to older children. Thus the oldest children in our sample might have been better able to represent these relationships, and better able to recognize that equal allocations to animal types would require unequal allocations to boxes. A prediction arising from this idea is that adults might show less partition dependence, or even none, in the same task.

Replication and extension of this work with new populations and additional childappropriate paradigms will be an important goal for future research. Although we think it is likely that this paradigm does have good internal validity, there are some potential limitations. In order to keep the task brief and understandable for very young children, we minimized verbal instructions and explanations. As a result, it is possible that children's interpretations of the task did not necessarily match ours, or that younger and older children might have made different interpretations. For example, did the children indeed understand what was implied by our stimuli and verbal instructions: that the animal types depicted on the double box would have to share the 
food placed in their box, but the animal types depicted on the single boxes would not? Another potential limitation is that children were given the fairness cue ("Alex wants to be fair") early in the instructions, and then the final instruction they received was "Ready to help Alex give out the food? You can put it in the boxes however you like." A possible objection to this script is that older children might be more likely to remember that first instruction to be fair (e.g. presumably to allocate evenly to the animal types, though this was of course never explicitly stated), while younger children might forget (and perhaps be less influenced by the fairness instruction). If this were true, it could provide a different explanation of our finding of a larger partition dependence effect in younger children: younger children would simply be allocating equally across boxes because they had forgotten that they were supposed to allocate "fairly." Our view is that "you can put it in the boxes however you like" is unlikely to override children's intrinsic tendency toward fair sharing along with our explicit instruction about fairness. However, the possibility should be explored in future studies.

Influences on children's decision making are important to understand, both in order to specify the developmental course of decision processes and for their potential practical implications. Children, like adults, make decisions with consequences, and children's decisions, like adults', are potentially subject to partition dependence. They may choose weekly schoolbased experiences, selecting from menus of enrichment options, arts, music, physical activities, and so on. Older children may make decisions about specialization in school subjects, or choices related to healthcare options. Even very young children may choose snacks and meals, or play options - perhaps differing by gender - that have later consequences for spatial and mathematical skills (e.g. Jirout \& Newcombe, 2015). Such decisions could have longer-term consequences for health, nutrition, school success, avocations, and careers. An understanding of the development of partition dependence may be helpful in avoiding choice architectures that unintentionally nudge children in less desirable directions, and could be leveraged to help caregivers nudge them toward better decisions (e.g. Johnson et al., 2012; Thaler \& Sunstein, 2008).

In addition to providing the first evidence of partition dependence in childhood, this work sets the stage for future research on the influence of partitions on reasoning across the lifespan. The current study leaves unanswered the important questions of whether other classes of decisions in children's lives would also lead to partition-dependent behaviors, or whether children's real-world allocations or choices are also subject to partitioning effects. Moreover, different classes of underlying psychological mechanisms have been proposed as leading to partition dependent behavior under differing conditions (Fox, Bardolet et al., 2005). The work in this area is still in very early stages. A developmental perspective on these questions may elucidate the relative contributions of different potential mechanisms, and may articulate their emergence with age. 


\section{References}

Bardolet, D., Fox, C.R., \& Lovallo, D. (2011). Corporate capital allocation: A behavioral perspective. Strategic Management Journal, 32, 1465-1483.

Benartzi, S., \& Thaler, R. H. (2001). Naive diversification strategies in defined contribution saving plans. The American Economic Review, 91, 79-98.

Blake, P.R., \& Rand, D.G. (2010). Currency value moderates equity preference among young children. Evolution and Human Behavior, 31, 210-218.

Chernyak, N., Sandham, B., Harris, P. L., \& Cordes, S. (2016). Numerical cognition explains age-related changes in third-party fairness. Developmental Psychology, 52, 1555-1562.

Echelbarger, M., \& Gelman, S. (2017). The value of variety and scarcity across development. Journal of Experimental Child Psychology, 156, 43-61. Fehr, E., Bernhard, H., \& Rockenbach, B. (2008). Egalitarianism in young children. Nature, 454, 1079-1083.

Fox, C. R., Bardolet, D. \& Lieb, D. S. (2005). Partition dependence in decision analysis, resource allocation, and consumer choice. Chapter in R. Zwick, R. \& A. Rapoport (Eds.), Experimental Business Research. Volume III (Dordrecht, The Netherlands: Kluwer).

Fox, C. R., \& Levav, J. (2004). Partition-edit-count: Naïve extensional reasoning in conditional probability judgment. Journal of Experimental Psychology: General, 133, 626-642.

Fox, C. R., Ratner, R. K., \& Lieb, D. S. (2005). How subjective grouping of options influences choice and allocation: Diversification bias and the phenomenon of partition dependence. Journal of Experimental Psychology: General, 134, 538-551.

Fox, C. R., \& Rottenstreich, Y. S. (2003) Partition priming in judgment under uncertainty. Psychological Science, 14, 195-200.

Jirout, J.J., \& Newcombe, N.S. (2015). Building blocks for developing spatial skills: Evidence from a large, representative U.S. sample. Psychological Science, 26, 302-310.

Johnson, E. J., Shu, S. B., Dellaert, B. G. C., Fox, C., Goldstein, D. G., Haübl, G., \& Weber, E. U. (2012). Beyond nudges: Tools of a choice architecture. Marketing Letters, 23, 487-504.

Lakshminarayanan, V.R., Chen, M.K., \& Santos, L.R. (2011). The evolution of decision-making under risk: Framing effects in monkey risk preferences. Journal of Experimental Social Psychology, 47, 689-693.

Levin, I. P., \& Hart, S. (2003). Risk preferences in young children: Early evidence of individual differences in reaction to potential gains and losses. Journal of Behavioral Decision Making, 16, 397-413.

LoBue, V., Nishida, T., Chiong, C., DeLoache, J. S., \& Haidt, J. (2009). When getting something good is bad: Even three-year-olds react to inequality. Social Development, 20, 154-170.

Martin, J. M., \& Norton, M. I. (2009). Shaping online consumer choice by partitioning the web. Psychology \& Marketing, 26, 908-926.

Messick, D. M. (1993) Equality as a decision heuristic. In J. Baron \& B. A. Mellers (Eds.), Psychological perspectives on justice. Theory and applications (pp. 11-31). New York: Cambridge University Press.

Nguyen, S. P., \& Girgis, H. (2014). Classification and cross-classification in children. In C., Ruoling (Ed.), Cognitive Development: Theories, Stages and Processes and Challenges (pp. 17-32). New York: Nova Science Publishers. 
Read, D., Antonides, G., van den Ouden, L., \& Trienekens, H. (2001). Which is better: Simultaneous or sequential choice? Organizational Behavior and Human Decision Processes, 84, 54-70.

Read, D., \& Loewenstein, G. (1995). Diversification bias: Explaining the discrepancy in variety seeking between combined and separated choices. Journal of Experimental Psychology: Applied, 1, 34-49.

Reyna, V.F., \& Ellis, S.C. (1994). Fuzzy-trace theory and framing effects in children's risky decision making. Psychological Science, 5, 275-279.

Reichelson, S., Zax., A., Bass, I., Patalano, A., \& Barth, H. (2017). Partition dependence in consumer choice: Perceptual groupings do not reliably shape decisions. Psychonomic Bulletin \& Review. doi: 10.3758/s13423-017-1326-4.

Santos, L.R., \& Rosati, A.G. (2015) The evolutionary roots of human decision making. Annual Review of Psychology, 66, 13.1-13.27.

Schlottmann, A., and Tring, J. (2005). How children reason about gains and losses: framing effects in judgement and choice. Swiss Journal of Psychology, 64, 153-171.

See, K. E., Fox, C. R., \& Rottenstreich, Y. S. (2006) Between ignorance and truth: Partition dependence and learning in judgment under uncertainty. Journal of Experimental Psychology: Learning, Memory, and Cognition, 32, 1385-1402.

Simonson, I. (1990). The effect of purchase quantity and timing on variety seeking behavior. Journal of Marketing Research, 32, 150-162.

Simonson, I., \& Winer, R. S. (1992). The influence of purchase quantity and display format on consumer preference for variety. Journal of Consumer Research, 19, 133-138.

Smith, C. E., Blake, P. R., \& Harris, P. L. (2013). I should but I won't: Why young children endorse norms of fair sharing but do not follow them. PloS One, 8, e59510.

Sonneman, U., Camerer, C. F., Fox, C. R., \& Langer, T. (2013). How psychological framing affects economic market prices in the lab and field. Proceedings of the National Academy of Sciences, 110, 11779-11784.

Tannenbaum, D., Fox, C. R., \& Goldstein, N. J. (n.d.) Partitioning menu items to nudge singleitem choice. Working paper.

Tannenbaum, D., Doctor, J. N., Persell, S. D., Friedberg, M. W., Meeker, D., Friesema, E. M., Goldstein, N. J., Linder, J. A., \& Fox, C. R. (2015). Nudging physician prescription decisions by partitioning the order set: Results of a vignette-based study. Journal of General Internal Medicine, 30, 298-304.

Thaler, R., \& Sunstein, C. (2008) Nudge: Improving decisions about health, wealth, and happiness. Yale University Press, New Haven, CT.

Weber, M., Eisenführ, F. \& von Winterfeldt, D. (1988). The effects of splitting attributes on weights in multiattribute utility measurement. Management Science, 34, 431-445. 
RUNNING HEAD: Partition dependence

Appendix A

Example script for one condition (Tigers+Leopards/Cheetahs/Panthers)

"Would you like to play a game with me? This is my friend Alex! [show puppet] Alex has some food to give to animals at the zoo. [Show circular wooden tokens in small box]. Alex wants to be fair. Can you help Alex give out the food?"

[Experimenter always starts putting on cats at single box and ends at double box.]

Single Box: [Take a cat from behind the boxes] "Hmm...[look perplexed] why don't we put the food for all of the Panthers [motion to the cat] in this box." [velcro cat to box]

Single Box: [Take a cat from behind the boxes] "Hmm...[look perplexed] why don't we put the food for all of the Cheetahs [motion to the cat] in this box." [velcro cat to box]

Double Box: [Take two cats from behind the boxes] "Hmm...[look perplexed] why don't we put the food for all of the Leopards and all of the Tigers [motion to the cats] in this box." [velcro cats to box]

"Ready to help Alex give out the food? [Give box containing tokens to child.] You can put it in the boxes however you like." 